\title{
Research on Tangible Acoustic Interface and Its Applications
}

\author{
Tzren-Ru Chou \\ Department of Graphic Arts and Communication \\ National Taiwan Normal University \\ Taipei City, Taiwan (R.O.C) \\ trchou@ntnu.edu.tw
}

\author{
Jen-Chieh Lo \\ Department of Graphic Arts and Communication \\ National Taiwan Normal University \\ Taipei City, Taiwan (R.O.C) \\ turkeyworld64@gmail.com
}

\begin{abstract}
Tangible acoustic interface has potential to become a high value approach for interaction due to its inexpensive and easy-to-implement characteristics. The signal captured by acoustic sensors contains large amount of information, which can be applied to several fields, such as gesture detection, music installations and information transmission. Three major techniques for developing tangible acoustic interfaces are acoustic holography, time delay of arrival and location pattern matching. In this article, we describe each of these techniques, making an overview of their pros and cons, and discuss about the future research of tangible acoustic interface.
\end{abstract}

Keywords-Tangible Acoustic Interface; Acoustic Holography; Time Delay of Arrival; Location Pattern Matching; acoustic interaction

\section{INTRODUCTION}

Over the past 20 years, there have been many changes in Human-Computer Interaction (HCI). Traditional HCI systems are single-user and task-oriented. However, emerging HCI systems have a tendency to become multimodal and embedded. Thereby, they allow people to interact with them more naturally [1]. Smart environment is an emerging concept defined by Mark Weiser which is described as "a physical world that is richly and invisibly interwoven with sensors, actuators, displays, and computational elements, embedded seamlessly in the everyday objects of our lives and connected through a continuous networks" [2]. When the environment itself becomes the interface, everyday objects are turned into interactive ones. People go about their daily life and carry out their tasks in a smart and unobtrusive way while the computing technologies are there, disappearing into the background, to support them transparently. To reach this vision of ambient intelligence and pervasive computing, tangible acoustic interface is a good way for people to make interaction with daily objects in the most natural and intuitive manner.

The name of Tangible Acoustic Interface (TAI) comes from The EU-IST Project TAI-CHI (Tangible Acoustic Interfaces for Computer Human Interaction, http:// www.tachi.cf.ac.uk), funded by the European Commission since 2003. The goal of TAI-CHI project is to combine ordinary objects and surface with sensing technology, thereby turning any solid surfaces or objects into tangible interfaces. To put it another way, TAI uses microphones or accelerometers as vibration sensors to capture tactile information. By analyzing vibration, it is possible to determine where and how the object is touched, which could provide a complete description about the tangible interaction. TAI takes advantage of sound originated in air or in solids respectively during interaction with any item, while other touch technologies such as infrared grids and resistive touchscreen might need extra mechanical or electronic devices to support interaction. Further, all the sensing part can be hidden behind the surface or inside the object. This can reduce manufacturing costs and let the application extend into more fields.

The central purpose of this study is to discuss the major techniques of TAI, and make a preliminary exploration for its applications. In addition, we also make suggestions for future research in relation to the shortcomings of TAI. The reminder of this paper is organized as follows. Section II focuses on three major techniques used for developing TAI. In Section III, several common applications using TAI technology are described and classified by their purposes. Finally, the pros and cons of TAI are discussed and suggestions are made for future research.

\section{TECHNIQUES FOR TANGIBLE ACOUSTIC INTERFACES}

Principally, techniques used in tangible acoustic interfaces for detecting interactions can be grouped into two main families, active and passive. In active mode, signal detection is made based on the evaluation of the acoustic energy, which is absorbed at contact points of the object surface, when the object is excited with ultrasound. This kind of techniques is regarded as Acoustic Holography.

On the other hand, techniques in passive mode differ from active one. They rely on analyzing acoustic vibration generated by tapping or moving a finger on the surface of an object. Time Delay of Arrival (TDOA) and Location Pattern Matching (LPM) are two techniques laying the foundations of passive mode for TAI development.

\section{A. Acoustic Holography}

The main advantage of holography is that it can produce vast information of holograms. Three-dimensional wave field can be reconstructed from two-dimensional photosensitive surfaces using the saving phase information. Just like making an optical holography, the basic of making an acoustic holography is to measure cross-spectra between a set of reference transducers and the hologram microphones over a plane close to the radiating surface [3]. Using these measured cross-spectra, a complete three-dimensional description of the sound field can be obtained, e.g. sound 
intensity, particle velocity, sound pressure, sound power, radiation pattern, ... and the location of the sound source.

Because acoustic holography can only propagate to a surface that is parallel to the measured surface, researchers in Institute for Mechanical Engineering (IMW) investigate a method using sensors contacted on an object directly. This method is based on Acoustic Holography and uses ICP accelerometers arranged along a line, mounted on a wooden writing table as acoustic sensors [4]. However, propagation velocity is not constant in-solids. It still depends on the material. Therefore, it is essential to choose the most useful frequency for identification, and only singular events can be observed with this method currently.

\section{B. Time Delay of Arrival (TDOA)}

The method, Time Delay Estimation, has plenty of applications as diverse as radar, sonar, seismology, geophysics, ultrasonic, and communications for detecting, identifying, and localizing radiating sources. It can also be used as a major technique for developing TAI. As we mentioned above, when tactile interactions take place, vibration will be generated on the object surface and the corresponding signal is acquired through contact sensors (piezoelectric sensors or microphones). The principle of TDOA is based on analyzing and comparing the vibration detected by a number of sensors. When an interaction occurs on the surface of the structure, the time delay of acoustic wave arrival at each of sensing elements will be recorded. By using known propagation velocity, the distance between sensors and source position could be calculated and easily determined by a computer.

Beside performing well and having the advantage of quick response and simple computation, TDOA technique is suitable for identifying arbitrary locations within the working area defined by sensors boundary [5, 6]. However, the performance of TDOA-based solutions critically depends on accuracy and robustness of time delay estimation. When the tangible object becomes less homogenous, TDOA technique may not work properly because wave velocity in nonhomogenous medium is not equal in all directions and may result in errors on location detection [5]. The other disadvantage is that it needs many sensors, which might result in complicated hardware configuration.

\section{Location Pattern Matching (LPM)}

The principle of LPM technique is based on identifying the signal of interaction and comparing it with a pre-recorded signal database. The acoustic pattern received from a sensor can be regarded as the superposition of different localized signal components with different frequencies, phases and reflections in an object. By comparing received signal with a pre-recorded and pre-known signal database, we can easily use cross-correlation analysis to determine acoustic information such as source location from the best match with pre-recorded signal in the templates.

Differing from TDOA, LPM has the advantage of simple hardware configurations, as usually only one sensor is needed [7]. LPM also has the feature of noise reduction due to the mean filter effect in pattern matching by using cross-

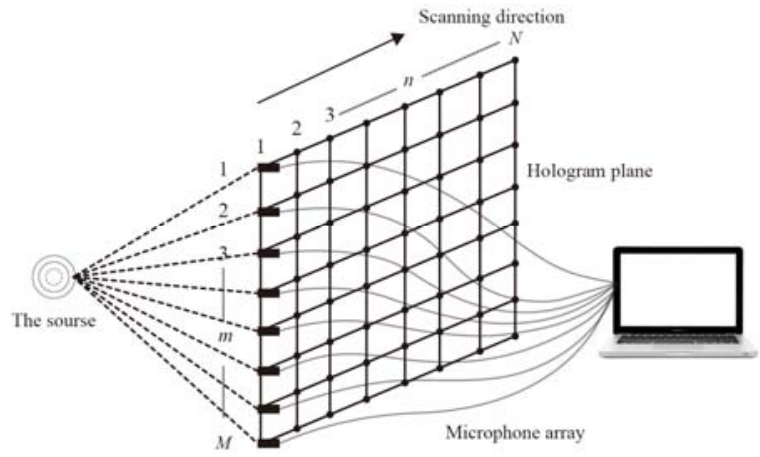

Figure 2. Acoustic Holography Diagram.

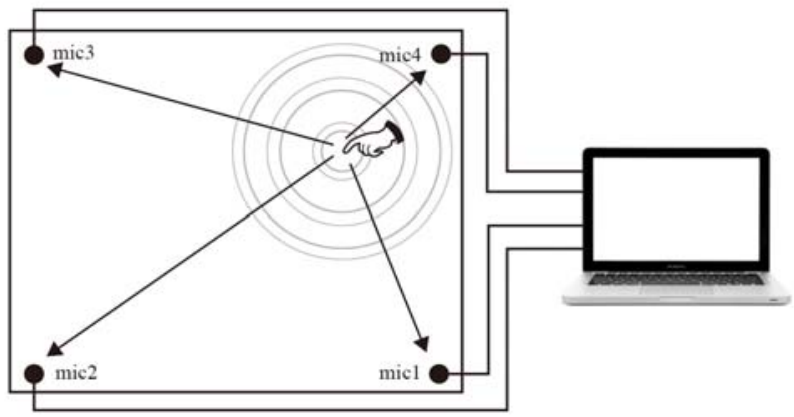

Figure 3. Time Delay of Arrival Diagram.

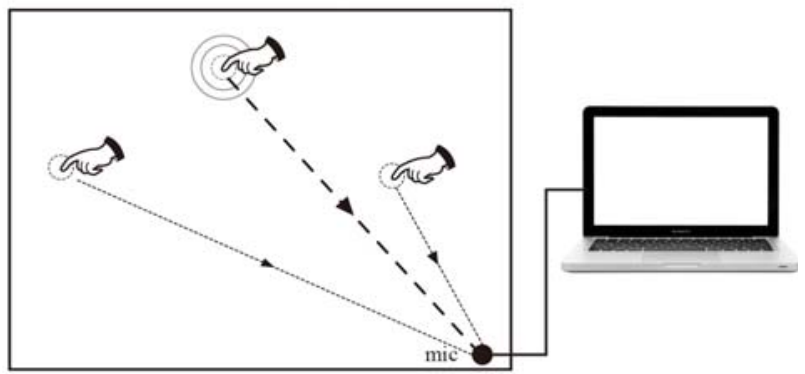

Figure 1. Location Pattern Matching Diagram.

correlation method. The drawback of the LPM is that its performance is slow and the computation is complicated because each renewed signal after interaction needs to be compared with saved tracks. 


\section{APPLICATIONS}

Recently, studies on transforming daily life objects into TAI have been increasing sharply over the past few years, and several interactive applications based on acoustic signal detection have been made. Here we describe several common applications and classify them into two different types according to their purposes.

\section{A. As Musical Installations}

"Sound Rose" is an interactive music table that consists of a touch sensitive table with images projected from the ceiling [9]. When users touch the table, rose-like graphics are displayed at the point of contact and the raw sound created by the interaction with the table is processed in real-time in order to produce more elaborated sound sequences. The interaction is based on the continuous touch-tracking algorithm enhanced expressiveness for the sonic and visual feedback. By using the dispersion property of in-solid acoustic wave propagation, a daily wooden table can be transformed into a musical and visual instrument easily.

The other example is called "Music Wall" which uses taps on a hard surface as the mode of interaction between the user and the information device [10]. The installation is implemented as a digital music player and constructed by small acoustic sensing modules for tracking location of taps or the rhythm of tap sequences.

\section{B. As Methods for Identification}

TAI researchers have explored many approaches for using TAI to encode or decode unique information. Gesture recognition is a commonly used application for TAI. The "Scratch Input" technique uses a microphone situated on wall or table surface to listen to the "scratching" sound and classify the gestures (taps, swipes and shapes) performed on the surface [11]. Sonically Enhanced Touch also use a microphone attached to an interactive surface in order to differentiate taps from different parts of fingers and allow detecting gestures on the bezel or side to become possible [12]. These works add sound as a new dimension that can provide more expression to user defined gestures.

In addition, “Acoustic Barcodes” provides another new way for information retrieval [13]. An Acoustic Barcode consists of a series of notches cut into a smooth host material. Just using a single, inexpensive contact microphone attached to surface to capture the waveform, system will decode the sound and retrieve information hidden inside the notches. Acoustic Barcodes are inexpensive and durable, and more importantly, they can be incorporated in a wide variety material.

\section{DISCUSSION}

Each of the different techniques that we described in Segment II has its own advantages and disadvantages. It is very important to take an appropriate method when we start to develop a tangible acoustic interface. Therefore, we give an overview of pros and cons of three TAI techniques in Table 1.
TABLE I. Pros AND CONS OF THREE TANGIBLE ACOUSTIC INTERFACE TECHNIQUES

\begin{tabular}{|c|c|c|}
\hline & Pros & Cons \\
\hline $\begin{array}{l}\text { Acoustic } \\
\text { Holography }\end{array}$ & $\begin{array}{l}\text { Ability of tracking several } \\
\text { points of contacts } \\
\text { simultaneously and } \\
\text { continuously. }\end{array}$ & $\begin{array}{l}\text { An array of contact } \\
\text { microphones attached to } \\
\text { the surface of the object } \\
\text { is necessary. }\end{array}$ \\
\hline $\begin{array}{l}\text { Time Delay } \\
\text { of Arrival }\end{array}$ & $\begin{array}{l}\text { Fast response and } \\
\text { simple computation. }\end{array}$ & $\begin{array}{l}\text { 1. Only applying on uniform } \\
\text { surfaces of } \\
\text { homogeneous material } \\
\text { (glass, PVC or metallic } \\
\text { plates). } \\
\text { 2. Need more sensors for } \\
\text { localizing (two sensors } \\
\text { for mono-dimensional } \\
\text { detection, and four for bi- } \\
\text { dimensional detection) }\end{array}$ \\
\hline $\begin{array}{l}\text { Location } \\
\text { Pattern } \\
\text { Matching }\end{array}$ & $\begin{array}{l}\text { 1. Only one or few } \\
\text { sensors are } \\
\text { necessary. } \\
\text { 2. Compatible with the } \\
\text { largest variety of } \\
\text { objects, even with } \\
\text { complex shapes. }\end{array}$ & $\begin{array}{l}\text { 2. Relatively big delay in the } \\
\text { response. }\end{array}$ \\
\hline
\end{tabular}

To those applications we described in Segment III, we can find most of acoustic interactive installations focus on the applications of localization. It is because acoustic technology is very good to obtain position information but not for identification [14]. Moreover, most of the TAI methods can only deal with singular events at a time, while having an ability to separate concurrent interaction and sophisticated gestures is just necessary for HCI systems to allow people to interact with machine in natural ways [4][11]. Accuracy and reliability are the key points for successful HCI systems. Factors, such as ambient noise, which might let interaction become ineffective, should be taken into consideration carefully during TAI development.

We believe that acoustic interactive systems have potential to become as popular as visual interactive system because of the enormous amount of information carried by acoustic signal. In order to get better interaction between human and machine by using TAI, it requires not only accurate localization but also refined characterization and interpretation of the interaction. Using different kind of signal processing techniques to associate with audio identification could also provide more information and smoothen the interaction [14]. For instance, we could use both acoustic and optical technologies for interaction detection, or using algorithm for audio signal separation to reduce the effect of ambient noise and solve the problem of detecting concurrent signal.

Further research could focus on following issues: exploring complicated sound gestures; separating concurrent events by using blind audio signal separation; enhancing the interaction accuracy by means of multi-sensory signal processing; and developing TAI with multi-sensory feedback.

\section{REFERENCES}

[1] R. Poppe, R. Rienks, and B. v. Dijk, "Evaluating the future of HCI: challenges for the evaluation of emerging applications," in Artificial Intelligence for Human Computing. vol. 4451, T. Huang, et al., Eds.: Springer Berlin Heidelberg, 2007, pp. 234-250. 
[2] D. Cook and S. Das, Smart environments: technology, protocols and applications (Wiley Series on Parallel and Distributed Computing): Wiley-Interscience, 2004.

[3] Reference to LMS International, "Acoustic Holography”, URL:http://www.lmsintl.com/acoustic-holography

[4] W. Rolshofen, P. Dietz, G. Schäfer, "Innovative interface for humancomputer interaction," in Proceedings of the 9th International Design Conference DESIGN 2006, 2006, pp. 611-618.

[5] P. Polotti, M. Sampietro, A. Sarti, S. Tubaro, and A. Crevoisier, "Acoustic localization tactile interactions for the development of novel tangible interfaces," in Proc. of the 8th Int. Conference on Digital Audio Effects (DAFX-05), Madrid, Spain, 2005.

[6] D. T. Pham, et al., "Tangible acoustic interface approaches.," in Proceedings of IPROMS 2005 Virtual Conference., 2005, pp. 497502.

[7] J. E. N. Christensen and S. J. Godsill, "Bayesian classification of acoustical waveforms under environmental variability," in Applications of Signal Processing to Audio and Acoustics (WASPAA), 2011 IEEE Workshop on, 2011, pp. 281-284.

[8] D. T. Pham, et al., "Acoustic pattern registration for a new type of human-computer interface," in Proceedings of the IPROMs 2005 Virtual Conference, 2005.

[9] A. Crevoisier, C. Bornand, A. Guichard, S. Matsumura, and C. Arakawa, "Sound rose: creating music and images with a touch table," presented at the Proceedings of the 2006 conference on New interfaces for musical expression, Paris, France, 2006.

[10] C. Hu, K. Tung, and L. Lau, "Music wall: a tangible user interface using tapping as an interactive technique," presented at the Proceedings of the 8th Asia-Pacific conference on Computer-Human Interaction, Seoul, Korea, 2008.

[11] C. Harrison and S. E. Hudson, "Scratch input: creating large, inexpensive, unpowered and mobile finger input surfaces," presented at the Proceedings of the 21st annual ACM symposium on User interface software and technology, Monterey, CA, USA, 2008.

[12] P. Lopes, R. Jota, and J. A. Jorge, "Augmenting touch interaction through acoustic sensing," presented at the Proceedings of the ACM International Conference on Interactive Tabletops and Surfaces, Kobe, Japan, 2011.

[13] C. Harrison, R. Xiao, and Scott Hudson "Acoustic barcodes: passive, durable and inexpensive notched identification tags," presented at the Proceedings of the 25th annual ACM symposium on User interface software and technology, Cambridge, Massachusetts, USA, 2012.

[14] E. Mugellini, O. A. Khaled, S. Pierroz, S. Carrino, and D.Chabbi, "Generic framework for transforming everyday objects into interactive surfaces," in Human-Computer Interaction. Ambient, Ubiquitous and Intelligent Interaction. vol. 5612, J. Jacko, Ed.: Springer Berlin Heidelberg, 2009, pp. 473-482. 